\title{
Uma Ferramenta de Apoio ao Ensino de Cálculo com Realidade Aumentada
}

\author{
Lidiane T. Pereira ${ }^{1}$, Douglas C. B. Oliveira ${ }^{1}$, \\ Igor F. Couto ${ }^{1}$, Alessandreia M. de Oliveira ${ }^{1}$, Rodrigo L. S. Silva ${ }^{1}$ \\ ${ }^{1}$ Departamento de Ciência da Computação (DCC/ICE) \\ Universidade Federal de Juiz de Fora (UFJF) - Juiz de Fora - MG - Brazil
}

\begin{abstract}
This paper presents a tool to aid teaching of calculus for undergraduate courses using Augmented Reality. It is a quadric surface viewing application for Android platform that displays six quadric surfaces. The tools and techniques used in the development and the evaluation results of the tool by the academic community will also be presented.
\end{abstract}

Resumo. Este artigo apresenta uma ferramenta de apoio ao ensino da disciplina de Cálculo para os cursos de graduação utilizando Realidade Aumentada. Trata-se de um aplicativo de visualização de superfícies quádricas para a plataforma Android onde são exibidas seis superfícies quádricas. Serão apontadas também as ferramentas e técnicas aplicadas em seu desenvolvimento além dos resultados da avaliação da aplicação pela comunidade acadêmica.

\section{Introdução}

A educação é um campo com grande potencial para o desenvolvimento de aplicações de Realidade Aumentada (RA), pois a utilização adequada desta tecnologia tem o potencial de enriquecer os materiais didáticos, aumentando assim o interesse dos alunos nestes conteúdos e criando maior interação entre os mesmos. Ferramentas de RA na educação são desenvolvidas, em sua maioria, para desktop, o que algumas vezes desencoraja seu uso em salas de aula convencionais, ficando sua plena utilização restrita a laboratórios providos de computadores com configurações atuais e ao menos uma câmera por unidade.

Recentes avanços tecnológicos tornam cada vez mais acessíveis os dispositivos móveis como smartphones e tablets com alto poder de processamento e boas câmeras integradas por um baixo custo. Assim, a utilização de aplicativos de RA para estes dispositivos acaba sendo mais viável do que em sistemas baseados em desktop.

Neste artigo é apresentado um aplicativo de RA denominado EducAR - Quadrics, desenvolvido para a plataforma móvel Android, como apoio ao ensino de Cálculo na graduação. O objetivo é que os alunos tenham acesso à ferramenta de forma gratuita em seus próprios dispositivos para que possam utilizar, não somente em sala de aula, mas também em seus estudos individuais.

Este trabalho está assim organizado: a Seção 2 apresenta os trabalhos relacionados e a Seção 3 os conceitos de Realidade Aumentada. A Seção 4 mostra o sistema proposto e a Seção 5 uma análise qualitativa da primeira versão da ferramenta. Na Seção 6 são apresentados o planejamento, a execução e a análise de resultados quantitativos e qualitativos do estudo experimental realizado. Na Seção 7 foram apresentadas algumas conclusões sobre os dados coletados e uma comparação com os trabalhos relacionados. Por fim, a Seção 8 conclui o trabalho e apresenta propostas de trabalhos futuros. 
VI Congresso Brasileiro de Informática na Educação (CBIE 2017)

Anais do XXVIII Simpósio Brasileiro de Informática na Educação (SBIE 2017)

\section{Trabalhos Relacionados}

Os principais trabalhos publicados na área de Informática na Educação no Brasil estão presentes em uma das quatro fontes promovidas pela Comissão Especial de Informática na Educação (CEIE): Revista Brasileira de Informática na Educação (RBIE), Simpósio Brasileiro de Informática na Educação (SBIE), Workshop de Informática na Escola (WIE) e Congresso Brasileiro de Informática na Educação (CBIE). Os trabalhos relacionados à utilização de RA na Educação estão presentes em três destas fontes, RBIE, SBIE e CBIE, em publicações a partir de 2011 [Posada et al. 2016]. Diante disso, foram realizadas buscas por estes trabalhos com o intuito de identificar propostas relacionadas à EducAR Quadrics, também desenvolvidas para dispositivos móveis. Tais propostas são sumarizadas a seguir.

O aplicativo What Is Figure? é apresentado em [Oliveira et al. 2016] onde a RA é aplicada para apoiar o ensino de língua inglesa. A aplicação facilita a assimilação de palavras e melhora a aquisição de vocabulário para usuários que estudam o nível básico da língua. A aplicação mostra modelos em três dimensões (3D) do objeto real correspondente à palavra selecionada pelo usuário. A aplicação funciona em celulares Android e não necessita de redes móveis para ser executada. A interação existente entre o usuário e o conteúdo em RA consiste na visualização e na mudança dos modelos ao trocar a palavra selecionada. Neste trabalho não foi apresentada uma avaliação da ferramenta.

A aplicação proposta em [Cavalcante et al. 2016] auxilia crianças nos primeiros anos escolares na fixação do conteúdo lecionado. São exibidos modelos 3D de animais, bem como o nome dos mesmos em letras maiúsculas, representando imagens cotidianas vistas pelas crianças, que facilitam seu entendimento. No aplicativo, a criança tem acesso a uma lista de animais disponíveis e pode visualizar os modelos 3D alternando entre eles. O aplicativo funciona em celulares Android e não necessita de redes móveis para sua operação. Nenhuma avaliação da aplicação proposta foi apresentada.

Em [Fernandes et al. 2016] é apresentada uma aplicação de RA cujo objetivo é ajudar crianças com dificuldades no aprendizado escolar e na alfabetização, especialmente crianças autistas. A aplicação exibe em 3D números, letras, sílabas, palavras, animais e frutas a fim de facilitar o relacionamento da criança com esse conteúdo no mundo real. O usuário pode visualizar o conteúdo em RA e alternar entre as opções de conteúdo disponíveis. O aplicativo funciona em celulares Android e não precisa de redes móveis para sua operação. Uma avaliação da ferramenta foi proposta como atividade futura.

Em [Silva et al. 2015] é proposta uma aplicação para apoiar o ensino de Resistência dos Materiais nos cursos de Engenharia. Na aplicação, os alunos visualizam conteúdo 3D que complementa os roteiros de exercícios, facilitando a visualização das configurações dos objetos. A ferramenta foi desenvolvida para tablets e não precisa de redes móveis. Uma análise para verificar a aceitação da ferramenta foi efetuada com 50 alunos. Eles responderam um questionário com 18 perguntas. Os alunos foram positivos em relação à utilidade de RA na disciplina e às orientações passadas. Além disso, os alunos não estão acostumados a utilizar os recursos de RA, de acordo com suas respostas.

Em [Santos et al. 2013] é apresentado o projeto ALRA, uma engine onde as regras, as interações de usuários e toda a mecânica do jogo são processadas, para facilitar o desenvolvimento de jogos com RA no meio educacional. Como exemplo, foi cons- 
truído um jogo no qual o usuário deve se locomover no ambiente real do campus para cumprir as tarefas indicadas por meio de RA no aplicativo e ganhar pontos. É necessário que o dispositivo esteja conectado à Internet para que as missões sejam atualizadas. A engine permite que sejam criados jogos para os dispositivos que utilizam a plataforma Android. Vale mencionar que no artigo foi apresentado um exemplo de utilização mas sem avaliação experimental.

O sistema proposto no presente artigo foca em um conteúdo específico da Matemática para o ensino superior, as superfícies quádricas. Ele se difere dos citados por apresentar um aplicativo de RA para dispositivos móveis, com interface otimizada para celulares, propiciando um maior poder de investigação das superfícies estudadas dado que pode movimentar tanto o marcador quanto o dispositivo de visualização. Também pode, em tempo real, não apenas visualizar as superfícies, como alterar seus parâmetros e perceber as consequências das alterações. A ferramenta é gratuita e foi disponibilizada através da Play Store para usuários que possuam dispositivos com sistema Android. A avaliação desta ferramenta está detalhada nas Seções 6 e 7.

\section{Fundamentação teórica}

Realidade aumentada pode ser definida como um sistema que combina objetos reais e virtuais em um ambiente predominantemente real de forma interativa e em tempo real, podendo abranger todos os sentidos, incluindo tato, audição e olfato [Azuma et al. 2001].

Para o correto alinhamento dos objetos é necessário um sistema de rastreamento. Um sistema de rastreamento óptico baseado em marcadores pode ser construído através do uso de uma câmera simples, resultando em um sistema de baixo custo e fácil utilização. Um marcador fiducial foi o objeto real utilizado para calcular a posição na qual será inserido o objeto virtual. Para isso, a imagem do marcador é capturada através da câmera e algoritmos de visão computacional são aplicados para calcular sua posição e orientação.

Dispositivos como tablets e smartphones pertencem a classe dos handheld displays, isto é, são telas de LCD que possuem uma câmera acoplada onde são exibidas imagens do ambiente real. Objetos virtuais são desenhados nas telas dos dispositivos sobrepondo a imagem capturada do ambiente. Desta forma, o display funciona como uma camada transparente que proporciona ao observador a visão dos ambientes real e virtual combinados [Azuma et al. 2001]. Atualmente dispositivos móveis contam com alto poder de processamento e câmeras satisfatórias com baixo custo. Sendo assim, o desenvolvimento de aplicações de RA para esses aparelhos tem se tornado cada vez mais viável.

\section{Sistema Proposto}

\subsection{Arquitetura}

A EducAR - Quadrics foi desenvolvida com foco na plataforma Android. Para tanto, utilizou-se a biblioteca de RA denominada $A n d A R^{1}$. Esta biblioteca open source é baseada no ARToolkit e implementada em Java, que é a linguagem utilizada no desenvolvimento para a plataforma Android. A biblioteca é responsável pela identificação do marcador e cálculo da orientação e posição do objeto virtual. A renderização dos objetos virtuais é

\footnotetext{
${ }^{1}$ https://code.google.com/archive/p/andar/
} 
VI Congresso Brasileiro de Informática na Educação (CBIE 2017)

Anais do XXVIII Simpósio Brasileiro de Informática na Educação (SBIE 2017)

realizada separadamente por uma biblioteca gráfica. A plataforma Android traz, a partir da versão 2.3, uma implementação nativa da biblioteca OpenGL ES 2.0 (OpenGL for Embedded Systems). Trata-se de uma versão da biblioteca OpenGL otimizada para dispositivos móveis ${ }^{2}$. Visando a reprodutibilidade do aplicativo, disponibilizamos um diagrama representando a arquitetura de software da ferramenta desenvolvida neste trabalho ${ }^{3}$.

\subsection{Apresentação do Aplicativo}

A EducAR - Quadrics está disponível para download através da Play Store e é compatível com qualquer dispositivo com câmera integrada e sistema operacional Android 2.3 ou superior. Para cada superfície é exibido seu modelo 3D, sua equação com os parâmetros que podem ser alterados e uma barra de progresso onde o usuário altera os valores dos parâmetros. Na Figura 1, está ilustrado no canto superior direito a equação do Elipsoide e na parte inferior da imagem a barra de alteração do parâmetro selecionado (neste caso, o parâmetro $a$ ).

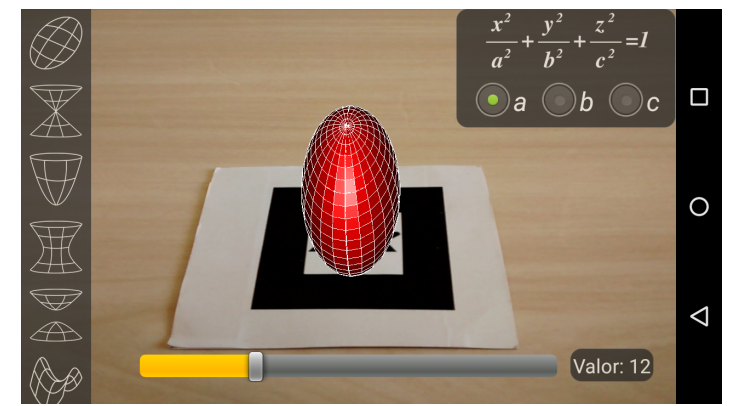

Figura 1. Interface de alteração de parâmetro da superfície Elipsoide.

\section{Estudo Preliminar}

Sistemas educacionais, como a EducAR - Quadrics, tem por objetivo auxiliar o processo de aprendizagem dos alunos. Inicialmente foi desenvolvida uma primeira versão da ferramenta e uma análise de caráter qualitativo foi realizada. O método escolhido foi a aplicação de questionário que é um método de baixo custo, fácil de ser aplicado e que efetivamente possibilita a obtenção de informações a respeito de interface e usabilidade [Root and Draper 1983]. Também foi levado em conta a massiva utilização dessa técnica em grande parte dos trabalhos encontrados na literatura, como os citados na Seção 2.

O questionário foi disponibilizado por meio do Google Forms e os usuários responderam questões sobre seus perfis, sobre o aplicativo e a utilização da RA no ensino de forma geral. Os participantes instalaram o aplicativo para verificar seu funcionamento e todos já haviam cursado ou estavam cursando Cálculo II, onde as superfícies quádricas são apresentadas. Participaram 35 pessoas dos cursos do Instituto de Ciências Exatas ou Engenharias. Quando questionados se consideravam que o aplicativo facilitaria a compreensão do conteúdo, 28 responderam "Sim", 6 "Talvez" e 1 "Não". A seguir, foram questionados se relacionavam ou não a dificuldade apresentada por outros colegas sobre determinados conteúdos à falta de ferramentas de apoio ao ensino. À essa pergunta 26 responderam "Sim", 6 "Talvez" e 3 "Não". Por fim, avaliaram o aplicativo quanto a interface, facilidade de uso e recursos oferecidos em uma escala de 5 níveis (Tabela 1).

\footnotetext{
${ }^{2}$ https://www.khronos.org/api/opengles/

${ }^{3}$ http: //bit.ly/ArquiteturaFerramenta
} 
VI Congresso Brasileiro de Informática na Educação (CBIE 2017)

Anais do XXVIII Simpósio Brasileiro de Informática na Educação (SBIE 2017)

Tabela 1. Avaliação da interface, facilidade de utilização e recursos oferecidos.

\begin{tabular}{|l|c|c|c|}
\cline { 2 - 4 } \multicolumn{1}{c|}{} & Interface & Utilizaçao & Recursos \\
\hline Muito Ruim & 0 & 0 & 0 \\
\hline Ruim & 0 & 0 & 0 \\
\hline Regular & 3 & 0 & 11 \\
\hline Bom & 9 & 6 & 13 \\
\hline Muito Bom & 23 & 29 & 11 \\
\hline
\end{tabular}

Após a análise das respostas, iniciou-se o desenvolvimento da segunda versão. Foi levada em conta a avaliação dos recursos oferecidos e foi criada a funcionalidade de alteração dos parâmetros das superfícies sugerida por participantes que usaram a primeira versão. Esta segunda versão foi usada no estudo experimental da seção seguinte.

\section{Estudo Experimental}

Neste estudo, pretende-se caracterizar a visualização de superfícies quádricas através de duas abordagens para obtenção de um esboço da superfície, do ponto de vista de usuários que já estudaram esse conteúdo. A primeira abordagem consiste na EducAR - Quadrics. A segunda abordagem consiste na utilização de material didático impresso contendo o esboço e as equações de cada uma das quádricas. O propósito desse estudo é, portanto, responder às seguintes questões de pesquisa:

- QP1 - A identificação da superfície e a criação de um esboço da mesma utilizando a EducAR - Quadrics como material de apoio é mais eficaz que a abordagem que utiliza somente o material impresso?

- QP2 - A identificação da superfície e a criação de um esboço da mesma utilizando a EducAR - Quadrics como material de apoio é mais eficiente que a abordagem que utiliza somente o material impresso?

\subsection{Definição e Planejamento}

Inicialmente um treinamento relacionado a superfícies quádricas foi elaborado. Também foi apresentada uma contextualização do estudo a partir de uma tarefa exemplo. Foram elaboradas três tarefas diferentes para cada uma das duas etapas do estudo. As seis tarefas apresentavam equações das principais superfícies quádricas para serem identificadas e esboçadas. Para a QP1, a quantidade de acertos na realização das tarefas foi utilizada. Já para a QP2, foi registrado também o tempo de execução de cada tarefa do estudo, em cada etapa. Antes da execução do estudo, um projeto piloto com esta mesma estrutura foi realizado com dois participantes cuja proposta era detectar possíveis problemas no material planejado para o estudo bem como na sua execução, permitindo que este material fosse aprimorado antes de sua utilização. Além disso, durante a execução houve um cuidado por parte dos aplicadores do experimento para isolar as tarefas e fornecer somente os dados e ferramentas necessários ao participante em cada etapa do experimento.

\subsection{Execução do Estudo}

Inicialmente o convite para participação do estudo foi enviado para aproximadamente 100 alunos dos diversos cursos da área de exatas da instituição, que já haviam cursado a disciplina Cálculo 2. Destes, 43 preencheram um questionário de caracterização disponibilizado online 4 sendo que, efetivamente, 37 convidados participaram do estudo, divididos

\footnotetext{
${ }^{4}$ http://bit.ly/form_caracterizacao
} 
VI Congresso Brasileiro de Informática na Educação (CBIE 2017)

Anais do XXVIII Simpósio Brasileiro de Informática na Educação (SBIE 2017)

em 3 sessões. Dado o número de participantes maior que 30 foi possível realizar uma análise estatística mais aprimorada [Juristo and Moreno 2013].

No início da sessão, os participantes preencheram um formulário de consentimento ${ }^{5}$. Em seguida receberam o treinamento e participaram da contextualização do estudo. Em cada uma das 3 sessões, a execução do estudo foi dividida em 2 etapas e os participantes em 2 grupos. As respostas do questionário de caracterização serviram para efetuar a divisão dos participantes em grupos mais homogêneos. O critério considerado foi o ano de ingresso no curso. Foi definido um quadrado latino de dois tratamentos (material impresso e EducAR - Quadrics). As 6 tarefas foram divididas em duas etapas, denominadas etapa $1^{6}$ e etapa $2^{7}$. O primeiro grupo executou a etapa 1 e suas tarefas utilizando a EducAR - Quadrics como abordagem apoio; enquanto o segundo grupo utilizou o material impresso. Em um segundo momento, os participantes receberam novas tarefas (etapa 2), que foram executadas com a abordagem diferente da utilizada na primeira etapa.

Dos 37 participantes, 15 utilizaram a EducAR - Quadrics como abordagem de apoio na etapa 1 e o material impresso na etapa 2 e 17 participantes utilizaram o material impresso na etapa 1 e a EducAR - Quadrics, na etapa 2. Em cada sessão, procurou-se manter o mesmo número de participantes para cada abordagem. No entanto, na sessão 1 quatro participantes não utilizaram a abordagem correta indicada na folha de atividades e foram descartados. Na sessão 2, um participante não registrou o tempo de duração de uma atividade e foi descartado. Desta forma, 32 participantes executaram as atividades de acordo com as orientações. Para finalizar, os participantes responderam um questionário de encerramento ${ }^{8}$ para a obtenção de informações acerca do estudo.

\subsection{Avaliação da Eficácia e da Eficiência}

Para esta avaliação foi utilizado o ambiente de software livre R e a IDE RStudio. Inicialmente foi realizado o teste de Shapiro-Wilk [Conover 1999] para verificar o pressuposto de normalidade dos dados com um intervalo de confiança de 95\%, isto é, $\alpha$-value $=0,05$ [Royston 1995]. Considerou-se que no estudo foi realizada uma única tarefa, que consistiu da identificação da superfície quádrica, através de abordagens diferentes e repetidas vezes, porém com o mesmo nível de dificuldade e o mesmo propósito em cada execução. A Tabela 2 apresenta os p-value obtidos após a realização dos testes. A suposição da normalidade foi violada para a variável Acerto nas duas abordagens, visto que $p$-value $<$ $\alpha$-value. Também foi violada para a variável Duração na abordagem Material Impresso. Conclui-se então que a amostra não possui distribuição normal.

Tabela 2. P-value para o teste de normalidade das variáveis Acerto e Duração.

\begin{tabular}{|c|c|c|c|}
\hline \multicolumn{2}{|c|}{ Acerto } & \multicolumn{2}{c|}{ Duração } \\
\hline EducAR - Quadrics & Material Impresso & EducAR - Quadrics & Material Impresso \\
\hline 0.005285 & 0.006796 & 0.611 & 0.00000009552 \\
\hline
\end{tabular}

Foi então realizado o teste não-paramétrico Mann-Whitney [Wilcoxon 1945] para duas amostras independentes a fim de realizar a análise estatística dos dados. Inicialmente

\footnotetext{
${ }^{5}$ http://bit.1y/TCLE_EducAR

${ }^{6}$ http://bit.1y/Atividades_Parte1

${ }^{7}$ http://bit.1y/Atividades_Parte2

${ }^{8}$ http://bit.ly/form_avaliacao
} 
VI Congresso Brasileiro de Informática na Educação (CBIE 2017)

Anais do XXVIII Simpósio Brasileiro de Informática na Educação (SBIE 2017)

foi testada a hipótese de que existia diferença entre a população que utilizou o aplicativo e a que não usou. Os dados do $p$-value obtidos foram Acerto $=0.7146$ e Duracao $=$ 0.2888. Assim não se pode afirmar que existe diferença estatística ( $p$-value $>\alpha$-value). Em seguida, foi testada a hipótese de que existia diferença entre os grupos de participantes e também não foi possível afirmar devido aos valores obtidos no teste (Tabela 3).

Tabela 3. Teste Mann-Whitney para as variáveis Acerto e Duração.

\begin{tabular}{|c|c|c|c|}
\hline \multicolumn{2}{|c|}{ Acerto } & \multicolumn{2}{c|}{ Duração } \\
\hline EducAR - Quadrics & Material Impresso & EducAR - Quadrics & Material Impresso \\
\hline 0.8777 & 0.2534 & 0.7658 & 0.8816 \\
\hline
\end{tabular}

A Tabela 4 mostra uma análise sobre a corretude da tarefa realizada para as duas abordagens. Apesar do valor final das pontuações ficar muito próximo para as duas abordagens, nota-se que a quantidade de certos foi maior quando utilizou-se o aplicativo.

Tabela 4. Distribuição de acertos pelo total de participantes em cada etapa.

\begin{tabular}{|l|c|c|c|c|}
\cline { 2 - 5 } \multicolumn{1}{c|}{} & \multicolumn{2}{c|}{ Parte 1 } & \multicolumn{2}{c|}{ Parte 2 } \\
\cline { 2 - 5 } \multicolumn{1}{c|}{} & EducAR - Quadrics & Material Impresso & EducAR - Quadrics & Material Impresso \\
\hline $\mathbf{1}$ & $57.8 \%$ & $54.9 \%$ & $52.9 \%$ & $44.4 \%$ \\
\hline $\mathbf{0 . 5}$ & $20 \%$ & $33.3 \%$ & $31.4 \%$ & $37.8 \%$ \\
\hline $\mathbf{0}$ & $22.2 \%$ & $11.8 \%$ & $15.7 \%$ & $17.8 \%$ \\
\hline
\end{tabular}

Na Figura 2 são apresentados boxplots das distribuições das variáveis duração e acerto. Percebe-se que os valores foram muito próximos para as duas abordagens.

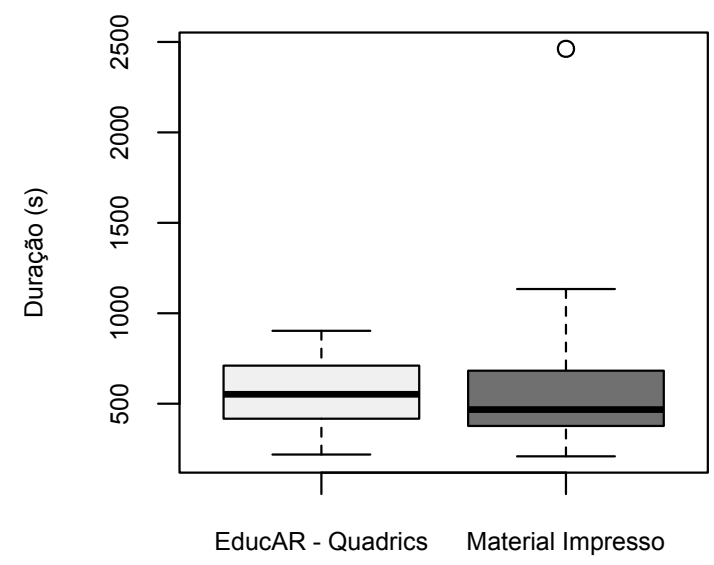

(a)

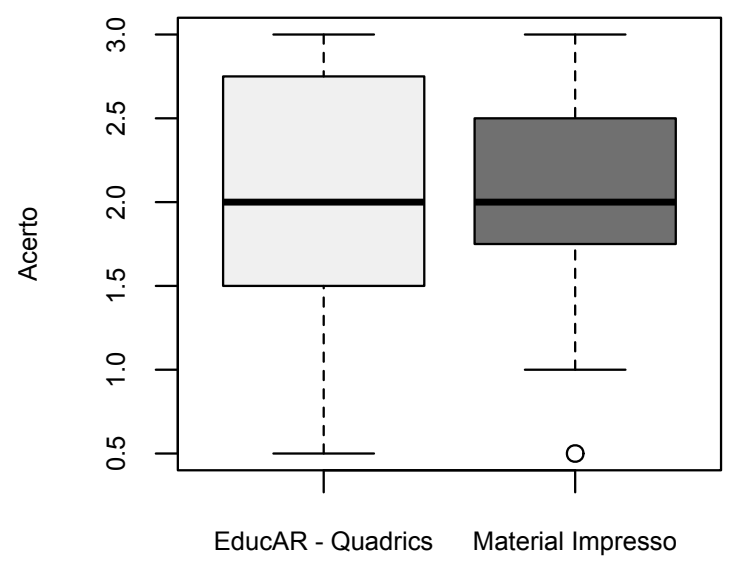

(b)

Figura 2. Em (a) a análise da variável duração e em (b) da variável acerto.

Após a realização dos testes e análise dos gráficos percebeu-se que diferente do esperado, os resultados foram muito parecidos. Isso pode ter decorrido do tamanho da amostra que apesar de relevante é ainda pequeno. O conhecimento prévio das superfícies também pode ter influenciado. Acredita-se que para alunos em processo de aprendizado do conteúdo exista uma diferença significativa no uso das duas abordagens combinadas. 
VI Congresso Brasileiro de Informática na Educação (CBIE 2017)

Anais do XXVIII Simpósio Brasileiro de Informática na Educação (SBIE 2017)

\subsection{Ameaças à Validade}

Durante o planejamento deste estudo, buscou-se minimizar ameaças que pudessem impactar ou limitar a validade dos resultados obtidos [Wohlin et al. 2000]. O estudo não foi executado em um único dia por todos os participantes. Não é possível confirmar que as circunstâncias eram as mesmas nas 3 sessões. Contudo, o mesmo roteiro e ambiente foram utilizados com a intenção de minimizar esta ameaça.

O estudo foi projetado para evitar o aprendizado dos participantes (com tarefas diferentes a cada etapa), mas não é possível confirmar que este efeito tenha sido totalmente eliminado. Além disso, o entendimento dos participantes sobre as questões é diretamente influenciado pela forma como foram elaboradas. A análise dos instrumentos utilizados (inclusive os formulários) a partir de um estudo piloto visou reduzir esta interferência.

Assumiu-se que os participantes seguiram as instruções e a ordem das atividades. Para minimizar esta ameaça, somente depois da conclusão da primeira etapa, é que foi apresentada a segunda. Adicionalmente, não é possível confirmar se a duração informada pelos participantes está correta. Finalizando, o tamanho da amostra é limitado. Desta forma, os resultados do estudo não são conclusivos: somente fornecem indícios. No entanto, o número de participantes superior a 30 é considerado alto [Juristo and Moreno 2013], o que aumenta a validade estatística das conclusões obtidas.

\subsection{Avaliação Qualitativa}

O formulário de encerramento foi dividido em quatro seções: Treinamento, Realidade Aumentada, Utilidade de aplicativos de RA e o aplicativo EducAR - Quadrics. O formulário foi respondido pelos 37 participantes de forma anônima e 33 possuíam dispositivo Android. Assim, os participantes descartados no estudo experimental foram considerados na análise apresentada na Tabela 5. Assim foi possível concluir em caráter qualitativo que a EducAR - Quadrics foi bem avaliada e do ponto de vista de alunos que já cursaram a disciplina tem potencial para apoiar o ensino do conteúdo das quádricas e incrementar o aprendizado.

Tabela 5. Resultados da avaliação qualitativa.

\begin{tabular}{|c|c|c|c|c|c|}
\hline Afirmação & DC & DP & $\mathbf{N} / \mathbf{I}$ & $\mathbf{C P}$ & $\mathbf{C C}$ \\
\hline As orientações do treinamento foram adequadas. & 0 & 0 & 0 & 3 & 34 \\
\hline Minhas dúvidas no treinamento foram adequadamente esclarecidas. & 0 & 0 & 2 & 0 & 35 \\
\hline Não é necessário treinamento para utilizar aplicativos de RA. & 1 & 8 & 2 & 20 & 6 \\
\hline Estou acostumado(a) a utilizar aplicativos de RA. & 6 & 8 & 4 & 13 & 6 \\
\hline O uso de aplicativos de RA pode melhorar a compreensão do conteúdo. & 0 & 0 & 0 & 9 & 28 \\
\hline Estou motivado(a) a utilizar os recursos de RA. & 0 & 0 & 5 & 14 & 18 \\
\hline Gostaria que as disciplinas do meu curso adotassem ferramentas de RA. & 0 & 0 & 3 & 12 & 22 \\
\hline Foi mais interessante identificar as superfícies utilizando a EducAR - Quadrics. & 0 & 2 & 1 & 9 & 25 \\
\hline A EducAR - Quadrics é útil para o estudo do conteúdo da disciplina Cálculo II. & 0 & 0 & 2 & 7 & 28 \\
\hline A ferramenta é intuitiva. & 0 & 1 & 2 & 25 & 9 \\
\hline O tamanho dos botões permite a correta manipulação da ferramenta. & 0 & 2 & 1 & 12 & 22 \\
\hline Os números e letras no aplicativo possuem tamanho adequado. & 1 & 5 & 0 & 7 & 24 \\
\hline O aplicativo apresenta uma interface visualmente agradável. & 0 & 0 & 2 & 22 & 13 \\
\hline
\end{tabular}


VI Congresso Brasileiro de Informática na Educação (CBIE 2017)

Anais do XXVIII Simpósio Brasileiro de Informática na Educação (SBIE 2017)

\section{Discussão}

Pode-se observar que os alunos consideram que existe potencial na ferramenta para apoiar o ensino de Cálculo, dado que a maioria das avaliações foi positiva nesse aspecto. Um número significativo de participantes inclusive relaciona a dificuldade apresentada por alguns alunos à falta de materiais de apoio. Isso pode ser consequência de que algumas ferramentas existentes necessitam de um hardware mais complexo para ser utilizada, como um computador que disponha de acesso à Internet e uma webcam, por exemplo. $\mathrm{O}$ uso do celular como plataforma torna o uso em sala de aula possível, visto que muitos dos alunos já possuem o hardware necessário à utilização e já estão familiarizados com o mesmo. Também foi possível constatar que os participantes avaliaram a facilidade de uso de forma muito positiva, o que pode estar relacionado ao fato de que a aplicação foi utilizada em celulares, plataforma onde os usuários já estavam adaptados à utilização.

Em relação aos recursos oferecidos, os usuários consideraram que apenas a visualização e troca das superfícies eram insuficientes, mas avaliaram positivamente a modificação em tempo real das superfícies virtuais. Esse recurso não é comum em outras aplicações de RA no meio educacional, sendo inclusive um diferencial da EducAR Quadrics.

A Tabela 6 compara as características da EducAR - Quadrics em relação aos demais trabalhos correlatos. Na primeira coluna são listados os trabalhos, na segunda o público alvo da aplicação. Na terceira coluna relacionamos o tipo de interação existente entre o usuário e os objetos virtuais apresentados nas aplicações. Na quarta coluna avaliamos se o aplicativo precisa de redes móveis para funcionar, Internet ou bluetooth por exemplo. Na última coluna indicamos se foi apresentada alguma avaliação do aplicativo.

Tabela 6. Comparação entre os trabalhos relacionados e a EducAR - Quadrics.

\begin{tabular}{|c|c|c|c|c|}
\hline Trabalho & Público-alvo & Interação & Rede & Avaliação \\
\hline [Oliveira et al. 2016] & Estudantes de nível básico de Inglês & V, T & Não & Não \\
\hline [Cavalcante et al. 2016] & Crianças dos primeiros anos escolares & V, T & Não & Não \\
\hline [Fernandes et al. 2016] & Crianças com necessidade especiais & V, T & Não & Não \\
\hline [Silva et al. 2015] & Graduandos em Engenharia & V & Não & Sim \\
\hline [Santos et al. 2013] & Desenvolvedores de jogos em RA & V & Sim & Não \\
\hline EducAR - Quadrics & Graduandos em Exatas e Engenharia & V, T, A & Não & Sim \\
\hline
\end{tabular}

\section{Conclusão}

Neste trabalho foi apresentado um visualizador de superfícies quádricas para dispositivos móveis como apoio ao ensino de Cálculo em cursos de graduação. O desenvolvimento teve como foco a plataforma Android e foi apoiado pela biblioteca AndAR. Foi apresentado à comunidade acadêmica uma versão preliminar do aplicativo e a avaliação desta versão foi feita através de um questionário. A partir da análise das respostas, concluise que os alunos acreditam que o uso de aplicativos baseados em RA pode facilitar a compreensão dos conteúdos estudados e possibilitar maior interesse nas disciplinas.

Inicialmente, um número significativo de alunos ressaltou que a EducAR - Quadrics deixa a desejar na quantidade de recursos. A maioria sugeriu que fossem implementadas novas funções além da visualização, como alteração de parâmetros das superfícies, 
VI Congresso Brasileiro de Informática na Educação (CBIE 2017)

Anais do XXVIII Simpósio Brasileiro de Informática na Educação (SBIE 2017)

desenho a partir de uma equação fornecida pelo usuário e rotação das superfícies sobre o marcador. Dessa forma, foi criada uma nova versão onde foi adicionada a função de alteração dos parâmetros das superfícies e os resultados da avaliação desta versão foram mais positivos. Diante da aceitação dos alunos, pretende-se realizar como trabalhos futuros melhorias na usabilidade e inclusão de novos recursos. Também pretende-se expandir o uso da RA na educação com aplicativos similares para outras áreas apresentadas na graduação.

\section{Referências}

Azuma, R., Baillot, Y., Behringer, R., Feiner, S., Julier, S., and MacIntyre, B. (2001). Recent Advances in Augmented Reality. IEEE Comput. Graph. Appl., 21(6):34-47.

Cavalcante, R. S., Fernandes, F. G., Lamounier, E., and Cardoso, A. (2016). Aplicação de Realidade Aumentada Móvel para Apoio ao Ensino de Crianças. Congresso Brasileiro de Informática na Educação, 5(1):691.

Conover, W. J. (1999). Practical nonparametric statistics. Wiley.

Fernandes, F. G., Oliveira, L. C. d., and Oliveira, E. C. d. (2016). Aplicação de Realidade Aumentada Móvel para Apoio à Alfabetização de Crianças com Autismo. Congresso Brasileiro de Informática na Educação, 5(1):1374.

Juristo, N. and Moreno, A. M. (2013). Basics of Software Engineering Experimentation. Springer Science \& Business Media. Google-Books-ID: iJTkBwAAQBAJ.

Oliveira, R. C. d., Silva, D., Fernandes, F. G., Oliveira, L. C. d., and Oliveira, E. C. d. (2016). Aplicativo de Aprendizagem Móvel utilizando Realidade Aumentada para Ensino de Língua Inglesa. Congresso Brasileiro de Informática na Educação, 5(1):731.

Posada, J. E. G., Buchdid, S. B., and Baranauskas, M. C. C. (2016). Informatics in education: what the works published in Brazil reveal. Brazilian Journal of Computers in Education, 24(01):142.

Root, R. W. and Draper, S. (1983). Questionnaires As a Software Evaluation Tool. In Proceedings of the SIGCHI Conference on Human Factors in Computing Systems, CHI '83, pages 83-87, New York, NY, USA. ACM.

Royston, P. (1995). Remark as r94: A remark on algorithm as 181: The w-test for normality. Journal of the Royal Statistical Society. Series C, 44(4):547-551.

Santos, O. L. d., Rafalski, J. d. P., and Menezes, C. S. d. (2013). Uma Game Engine para Aventuras Pedagógicas Locativas em Realidade Aumentada. Simpósio Brasileiro de Informática na Educação, 24(1):396.

Silva, J., Souza, F. d. F. d., Sedraz, L., and Ramos, J. L. C. (2015). Adoção de Realidade Aumentada no Ensino de Resistência dos Materiais. Congresso Brasileiro de Informática na Educação, 4(1):1198.

Wilcoxon, F. (1945). Individual comparisons by ranking methods. Biometrics bulletin, 1(6):80-83.

Wohlin, C., Runeson, P., Höst, M., Ohlsson, M. C., Regnell, B., and Wesslén, A. (2000). Experimentation in Software Engineering: An Introduction. Kluwer Academic Publishers, Norwell, MA, USA. 